Revista Calidad en la Educación Superior

Programa de Autoevaluación Académica

Universidad Estatal a Distancia, Costa Rica

ISSN 1659-4703

revistacalidad@uned.ac.cr

\title{
FACTORES QUE INFLUYEN EN LA PARTICIPACIÓN Y EL DESEMPEÑO ACADÉMICO DE LOS ESTUDIANTES QUE COMPITEN EN LA VII OLIMPIADA COSTARRICENSE DE CIENCIAS BIOLÓGICAS (OLICOCIBI).
}

FACTORS AFFECTING PARTICIPATION AND ACADEMIC PERFORMANCE OF STUDENTS COMPETE IN COSTA RICAN VII BIOLOGICAL SCIENCES

(OLICOCIBI) OLYMPICS

\begin{abstract}
José Pereira Chaves'jose.pereira.chaves@una.cr Ana Ulate Campos ${ }^{2}$ veronicaulatecampos12@yahoo.es

Stephanie Arroyo Jiménez ${ }^{3}$ stefnaz16@yahoo.com Universidad Nacional de Costa Rica
\end{abstract}

Volumen 6, Número 2

Noviembre 2015

pp. 1 - 22

Recibido: 7 de abril, 2015

Aprobado: 12 de junio, 2015

\footnotetext{
1 José M. Pereira Chaves, Doctor en educación, Máster en ciencias marinas, Licenciado en enseñanza de las ciencias.

2 Ana Ulate Campos. Licenciada en enseñanza de las ciencias. Universidad Nacional de Costa Rica.

3 Stephanie Arroyo Jiménez, Licenciada en enseñanza de las ciencias. Universidad Nacional de Costa Rica.
} 
Factores que influyen en la participación y el desempeño académico de los estudiantes que compiten en la vi olimpiada costarricense de ciencias biológicas (OLICOCIBI).

José M. Pereira Chaves, Verónica Ulate Campos, Stephanie Arroyo Jiménez.

\section{Resumen}

El objetivo de esta investigación fue analizar los factores que influyen en la participación y en el rendimiento académico de los estudiantes en la VII Olimpiada Costarricense de Ciencias Biológicas (OLICOCIBI). Como parte de la labor docente, es importante promover y facilitar el pensamiento científico en los estudiantes. Para ello es necesario que se motive y despierte en los docentes la curiosidad por plantearse preguntas de análisis, el pensamiento crítico y reflexivo, para que puedan comprender el mundo que los rodea y así superar las dificultades en el aprendizaje. La actitud científica debe fortalecerse, y ello depende de la disposición del educador para mantenerse informado de los avances científicos, así como del dominio de los conceptos y procesos que lo conducen, y así facilitar su adquisición. La Enseñanza de las Ciencias Biológicas utiliza técnicas que favorecen el desarrollo de las cualidades inherentes al espíritu científico designadas como procesos. Esta investigación se desarrolló en el marco de un proyecto de extensión durante el 2013, se enmarcó dentro del paradigma cualitativo, con un enfoque mixto, en donde se aplicaron 424 encuestas a estudiantes y 110 encuestas a profesores de biología, para su análisis se utilizó la estadística descriptiva, en donde se comprueba que la motivación y el gusto por la materia influyen en un buen rendimiento académico.

Palabras clave: Competencia cognitiva, olimpiada, rendimiento académico, educación, enseñanza de ciencias.

\section{Abstract}

The objective of this investigation was to analyze the factors that influence the participation and the academic performance of the students in the VII Costa Rican Olympics of Biological Sciences (OLICOCIBI). As part of the educational process, it is important to promote and to facilitate the scientific thought of the students. Therefore it is necessary to motivate and animate the curiosity of the teachers about asking questions of analysis, the critical and reflexive thought, so that they can understand the world that surrounds them and overcome the difficulties in learning. The scientific attitude must be strengthened, and it depends on the willingness of the educator to keep informed about the scientific advances, as well as about the domain of the concepts and processes that lead and facilitate their acquisition. Teaching Science uses techniques that favor the development of the qualities inherent to the scientific spirit designated as processes. This research was conducted as part of an extension project during 2013, using the qualitative paradigm, with a mixed approach, where 424 surveys were applied to students and 110 surveys to teachers of biology. The descriptive statistics was used for the analysis of the obtained information, which verifies that the motivation and the enthusiasm on biology influence in a good academic student's performance.

Keywords: Cognitive competence, Olympiad, academic performance, education, science teaching. 
Factores que influyen en la participación y el desempeño académico de los estudiantes que compiten en la vi olimpiada costarricense de ciencias biológicas (OLICOCIBI).

José M. Pereira Chaves, Verónica Ulate Campos, Stephanie Arroyo Jiménez.

La enseñanza y aprendizaje de las Ciencias implica una actitud abierta, investigativa, crítica y de reflexión, en la cual la visión del docente es un elemento clave en la educación. De esta manera, enseñar Ciencias es un desafío de las nuevas formas de abordar el pensamiento científico, la alfabetización científica y las competencias cognitivas son propuestas utilizadas en los últimos tiempos para fomentar la actitud científica de los estudiantes y de esta forma desarrollar un aprendizaje significativo (Veglia, 2007).

Para que el aprendizaje de las Ciencias sea significativo, la enseñanza de las mismas debe enfocarse en mejorar las capacidades de los educandos, y una alternativa son las competencias cognitivas que están relacionadas con el uso apropiado de las actitudes intelectuales y de las capacidades mentales para saber lo que se hace, con el manejo eficiente y eficaz de las técnicas, procesos, procedimientos y habilidades. Una competencia entonces podría ser de forma más sencilla y estructurada un saber ser, un saber pensar y un saber hacer (Lafrancesco, 2005).

Las competencias académicas, conocidas como Olimpiadas, están dirigidas a jóvenes estudiantes de educación secundaria y tiene como propósito estimular el estudio de las Ciencias Exactas y Naturales, así como descubrir jóvenes talentosos en estas disciplinas (Mora y Calderón, 2008).

Las competencias académicas representan a nivel científico una amplia tradición y relevancia en el mundo así como en Latinoamérica. Países como Argentina, Brasil y México llevan la delantera con sus excelentes niveles en organización, tanto para sus etapas nacionales como internacionales, lo que ha generado el interés de muchos jóvenes hacia áreas científicas (Pereira, Camacho y Muñoz, 2012). 
Factores que influyen en la participación y el desempeño académico de los estudiantes que compiten en la vi olimpiada costarricense de ciencias biológicas (OLICOCIBI).

José M. Pereira Chaves, Verónica Ulate Campos, Stephanie Arroyo Jiménez.

En Costa Rica con la idea de potenciar el conocimiento científico, tecnológico y sobre todo bioalfabetizar a los jóvenes del país, a inicios del 2007, la Ministra de Ciencia y Tecnología (MICIT), Dra. Eugenia Flores Vindas, mostró interés en que se organizará en Costa Rica las Olimpiadas de Biología, fue por ello que solicitó a las autoridades de la Universidad Nacional de Costa Rica por medio de la Facultad de Ciencias Exactas y Naturales y la Escuela de Ciencias Biológicas (ECB) la posibilidad de organizarla, los que acogieron la propuesta (Comisión Organizadora OLICOCIBI, 2009).

En ese mismo año se conformó un equipo interdisciplinario de académicos y estudiantes de la Escuela de Ciencias Biológicas, y con el apoyo de la Vicerrectoría de Extensión y dos estudiantes de Licenciatura, propusieron un trabajo de graduación denominado "I Olimpiada Costarricense de Biología", que fue un diagnóstico como propuesta evaluadora de la condiciones de trabajo, sirviendo como base para muchas de las actividades planteadas posteriormente en lo que se conoce hoy como OLICOCIBI. Para la I edición únicamente participaron estudiantes de undécimo año de secundaria, para 6 regionales educativas: Liberia, Pérez Zeledón, Limón, San José, Heredia y San Ramón (Pereira, Camacho y Muñoz, 2012).

De este modo, se precisaron y realizaron diversas acciones para el fomento de esta actividad, dentro de las cuales destacan: la reestructuración de un temario propio para la OLICOCIBI (basado en el temario del Ministerio de Educación Pública (MEP) y el alcance de éste en las instituciones de secundaria), se amplió la cobertura a nivel nacional y se aceptan estudiantes de todo los niveles desde séptimo hasta décimo año, además se incorporan otras instituciones de educación superior que tenía en sus actividades académicas la educación científica y biológica como la Universidad de Costa Rica (UCR) y Universidad Estatal a Distancia (UNED), por otra parte se implementa un programa de capacitaciones dirigidas a docentes en zonas rurales y urbanas, se regionaliza la aplicación de la 
Factores que influyen en la participación y el desempeño académico de los estudiantes que compiten en la vi olimpiada costarricense de ciencias biológicas (OLICOCIBI).

José M. Pereira Chaves, Verónica Ulate Campos, Stephanie Arroyo Jiménez.

prueba eliminatoria para llegar a todas las zonas rurales y finalmente se incursiona con la participación en competiciones internacionales (la Olimpiada Iberoamericana de Biología), lo que involucra un arduo proceso de preparación para estos competidores (Pereira, Camacho y Muñoz, 2012).

Cada una de esas acciones ha tenido importantes resultados, por lo que la OLICOCIBI, integrando la docencia, investigación y extensión, fue declarada de interés institucional en la Universidad Nacional (acuerdo SCU-159-2008; Gaceta №2 del 29 de febrero del 2008). Asimismo, por considerarse que amplía la cultura biológica de los jóvenes e impulsar las vocaciones científicas, acrecentando las habilidades, actitudes y valores del pensamiento racional y científico, fue declarada de interés educativo nacional por el Ministerio de Educación Pública (DVM-AC-00388-2011, 21 de marzo del 2011).

Desde entonces Costa Rica, al igual que otros países a nivel mundial cuenta, con la Olimpiada Costarricense de Ciencias Biológicas (OLICOCIBI), la misma tiene como objetivo general fortalecer y consolidar la formación de los y las estudiantes preuniversitarios que poseen interés y afinidad hacia las Ciencias Biológicas. OLICOCIBI se proyecta, como una actividad de gran alcance y significado, para el desarrollo de las Ciencias Biológicas en la educación secundaria y en la sociedad costarricense.

Estos eventos académicos no sólo pretenden fortalecer el conocimiento de los estudiantes, sino que también brinda espacios dirigidos a los docentes, para el mejoramiento del proceso de enseñanza y aprendizaje de las Ciencias Biológicas en secundaria. Durante las pruebas finales, se presentan espacios para compartir inquietudes y sugerencias sobre el evento lo cual conlleva a la formulación de mejoras para el mismo y enriquece y retroalimenta las actividades desarrolladas entre los actores de interés. 
Factores que influyen en la participación y el desempeño académico de los estudiantes que compiten en la vi olimpiada costarricense de ciencias biológicas (OLICOCIBI).

José M. Pereira Chaves, Verónica Ulate Campos, Stephanie Arroyo Jiménez.

Las olimpiadas Costarricense de Ciencias Biológicas, tiene un impacto en la sociedad costarricense, al considerársele como una oportunidad para el análisis y reflexión de los elementos conceptuales, procedimentales y actitudinales; entorno al desarrollo y participación de la actividad, ya que contribuye al mejoramiento de la actividad científica y tecnológica en el campo de las Ciencias Biológicas en el país (Camacho, 2011).

Como parte de la labor docente, se encuentra el promover y facilitar el pensamiento científico en los estudiantes. Para lograrlo es necesario que el docente despierte en los y las dicentes, la curiosidad por plantearse preguntas, el pensamiento crítico y reflexivo, para que puedan comprender el mundo que los rodea. El lograr este cambio suele ser un poco difícil, pues muchos educadores tienden a realizar una práctica educativa anticientífica, la misma se manifiesta según los propósitos y preocupaciones del docente, algunos tienen inquietudes como el lograr que los estudiantes alcancen los objetivos, aprueben los exámenes, constriñendo así su perspectiva de actuación al nivel del aula y dejando con ello de lado, otros contextos del trabajo docente no menos importantes como lo son la implementación del aprendizaje significativo de la Ciencia y el fortalecimiento de la criticidad de los educandos (Álvarez, 1998).

Por lo tanto, las OLICOCIBI, son competencias cognitivas donde el estudiante tiene la oportunidad de explorar y proyectar sus capacidades cognitivas y con ello, valorar su potencial en una determinada disciplina científica y así lograr identificar su futuro, así como su perfil de formación profesional, esto como una herramienta clave para su vida.

Tomando como base la normativa de OLICOCIBI se puede afirmar que la participación de la comunidad educativa, en competencias académicas orientadas a la promoción del conocimiento y aptitudes en la comprensión científica y humana de las ciencias biológicas, permite fortalecer la creación de una comunidad 
Factores que influyen en la participación y el desempeño académico de los estudiantes que compiten en la vi olimpiada costarricense de ciencias biológicas (OLICOCIBI).

José M. Pereira Chaves, Verónica Ulate Campos, Stephanie Arroyo Jiménez.

educativa-científica nacional, mediante encuentros regionales y nacionales, con la socialización y participación de docentes de biología, brindando espacios que aseguran la oportunidad de externar sus conocimientos, así como sus fortalezas y debilidades, gracias al análisis de las diversas pruebas teóricas y prácticas que desarrollan los estudiantes en el proceso (Comisión Organizadora OLICOCIBI, 2009).

Las Olimpiadas propician el intercambio de experiencias entre profesores, estudiantes, además se generan espacios de discusión entre los académicos de las instituciones organizadoras en donde se generan estrechas relaciones interpersonales entre los jóvenes participantes. En estas competencias se favorece la formación de futuros científicos con potencialidades que propicien el desarrollo científico y tecnológico del país a través de la motivación científica ya que se incentivan las vocaciones científicas (Mora y Calderón, 2008).

El presente trabajo tiene como objetivo identificar los factores que influyen en la participación y en el rendimiento académico de los estudiantes que compiten en la VII OLICOCIBI (2013). Lo que será un insumo importante para estudiantes y profesores con interés en estas competencias científicas, y, a la vez, presenta una visión general para los que organizan este tipo de eventos cognitivos en el quehacer científico.

\section{Aspectos Teóricos}

En Costa Rica, la Enseñanza de las Ciencias Naturales a nivel de secundaria se imparte de una manera teórica en su mayoría, por lo que se deja de lado la experimentación como herramienta didáctica imprescindible para la comprensión de los fenómenos científicos y biológicos (Pereira, 2010).

El abordaje de la biología, su enfoque particular y su naturaleza es formar otras disciplinas para la construcción de conceptos integrales, partiendo de la 
Factores que influyen en la participación y el desempeño académico de los estudiantes que compiten en la vi olimpiada costarricense de ciencias biológicas (OLICOCIBI).

José M. Pereira Chaves, Verónica Ulate Campos, Stephanie Arroyo Jiménez.

concepción del estudiante acerca de su realidad experimentada, por ello la biología como fuente del currículo, debe propiciar la educación y alfabetización científica (Pereira, 2010).

La enseñanza de la Biología se establece como una disciplina experimental, y el estudio de los fenómenos biológicos en las aulas de secundaria permite que los estudiantes pongan en práctica sus conocimientos teóricos y apliquen los pasos que caracterizan la investigación científica, lo que confiere un valor formativo que da a los estudiantes una mejor comprensión de la naturaleza de la ciencia y del conocimiento científico (Perales 2000).

En los procesos de enseñanza se debe visualizar al profesor como un mediador entre los contenidos y el aprendizaje gestado en el aula. Desde esta premisa, las Olimpiadas de Biología buscan contribuir a promover vocaciones científicas de los estudiantes sobre los procesos biológicos, las cuales se relacionan básicamente con los siguientes aspectos: planteamientos metodológicos que fundamentan la acción docente del profesor, criterios para la selección y secuencia de actividades de enseñanza, y por último, la importancia de los trabajos prácticos y de las actividades de resolución de problemas en la enseñanza de la Biología (Perales 2000).

El docente al ser el mediador, se torna un factor clave en la motivación de los estudiantes, la misma puede definirse, como el conjunto de elementos o factores que están activamente presentes, en su momento dado en la conciencia del ser humano y que configuran la fuerza psíquica y los mecanismos de estímulo que conducen a la acción (Orozco, 2011).

Existen factores que influyen de forma trascendental en el desarrollo del proceso de enseñanza y aprendizaje, entre los cuáles, la motivación es clave. La falta de motivación es un factor influyente en el rendimiento académico, ya que es la 
Factores que influyen en la participación y el desempeño académico de los estudiantes que compiten en la vi olimpiada costarricense de ciencias biológicas (OLICOCIBI).

José M. Pereira Chaves, Verónica Ulate Campos, Stephanie Arroyo Jiménez.

adquisición de nuevos aprendizajes, lo que anima precisamente al trabajo y el esfuerzo del alumno. Es posible que la motivación venga a ser la causa en definitiva de las conductas de aprendizaje y de rendimiento escolar.

La economía es un factor de cierta forma influyente en el desempeño escolar, es posible determinar que el origen social, la clase, la zona de residencia y ciertos aspectos culturales de las familias, son aspectos que explican las diferencias en el desempeño académico (Muñoz, Márquez, Sandoval, y Sánchez, 2004).

\section{Metodología}

La investigación se realizó considerando las siguientes fases.

Fase Preparatoria: En este periodo se recolectó la información a partir de los sujetos participantes. Por lo que se identificó a los actores de interés y se formuló un cuestionario para estudiantes que participaron en la fase eliminatoria (Junio 2013) y la final (Agosto 2013) de la VII OLICOCIBI, en la categoría A y a docentes tutores de cada uno de ellos durante la misma actividad. En esta etapa, también se identificaron los diversos factores que podrían afectar el rendimiento y la participación de los estudiantes, los mismos fueron tomados en cuenta en la elaboración de las encuestas utilizadas.

Trabajo de Campo: El cual se fue accediendo progresivamente a la información fundamental para su estudio. Esta fase consistió en la aplicación de los instrumentos a la población en estudio (Rodríguez, Gil y García, 1996). La misma se llevó a cabo en la fase eliminatoria, en las diferentes sedes regionales. Se aplicaron 463 encuestas (374 a estudiantes y 89 a docentes tutores) a nivel nacional y en agosto del 2013 se llevó a cabo la fase final en el Campus Omar Dengo y en la Estación de Ciencias Marino Costeras en Punta Morales donde se aplicaron 81 encuestas (60 a estudiantes y 21 a profesores). 
Fase Analítica: Se relacionaron los resultados obtenidos a partir de ambas fuentes de información (estudiantes y docentes), para compararse según las premisas preliminares. En esta etapa, se analizaron los resultados obtenidos en la aplicación de los instrumentos (encuestas). Aquí se comparó la información obtenida, y luego se realizó un análisis bibliográfico, con el fin de obtener un resultado consistente que le diera validez a la investigación sobre los factores que influyen en el éxito de esta competencia. Esta información se sistematizó con el uso del paquete estadístico SPSS (Statistical Product and Service Solutions) versión 17.0 para la tabulación de la información recolectada y Excel de Microsoft 2007 en la transformación a gráficos y tablas de los resultados obtenidos.

Las categorías de análisis del estudio fueron:

1. Los factores que influyen en la participación de los estudiantes en la categoría $A$ de la VII Olimpiada Costarricense de Ciencias Biológicas (OLICOCIBI).

2. Los factores que favorecen la clasificación y el buen rendimiento académico de los estudiantes en el proceso final de la Olimpiada de Ciencias Biológicas.

3. Las características del docente que promueve la participación de sus estudiantes a eventos científicos de competencias cognitivas.

Los objetos fuentes de estudio en esta investigación corresponden a una muestra de estudiantes de secundaria de las 21 direcciones regionales educativas del país, que participaron en la VII OLICOBICI tanto de la fase eliminatoria como en la etapa final y sus respectivos profesores (as) tutores (as).

\section{Principales Hallazgos}

De acuerdo con el análisis documental y los datos obtenidos en los diversos instrumentos se logró determinar que un factor que influye en la participación y en el buen rendimiento académico es el motivacional, el cual se detalla a continuación: 
Factores que influyen en la participación y el desempeño académico de los estudiantes que compiten en la vi olimpiada costarricense de ciencias biológicas (OLICOCIBI).

José M. Pereira Chaves, Verónica Ulate Campos, Stephanie Arroyo Jiménez.

\section{Factores Motivacionales}

Una de las causas que motivan la participación y el buen rendimiento académico en las OLICOCIBI lo que se muestra en el Cuadro 1.

\section{Cuadro1: Factores que motivaron la participación de los estudiantes en la VII OLICOCIBI según estudiantes y docentes. I y II etapa, agosto 2013.}

\begin{tabular}{l|c|c}
\hline $\begin{array}{l}\text { Factores que influyen } \\
\text { en la participación en } \\
\text { OLICOCIBI }\end{array}$ & $\begin{array}{c}\text { Según el estudiantado } \\
(\%)\end{array}$ & $\begin{array}{c}\text { Según los docentes } \\
(\%)\end{array}$ \\
\hline $\begin{array}{l}\text { Gusto propio por la } \\
\text { biología }\end{array}$ & 44 & 20,2 \\
\hline $\begin{array}{l}\text { Preparación para } \\
\text { Bachillerato }\end{array}$ & 33 & 27 \\
\hline Motivación docente & 22 & 49,4 \\
\hline Motivación familiar & 1 & 3,4 \\
\hline Total & $100 \%$ & $100 \%$ \\
\hline
\end{tabular}

Fuente: Elaboración propia.

El docente es el principal motivador de sus educandos y dicha motivación puede ser alcanzada a través de tres niveles: la extrínseca que está relacionada con las necesidades del tener, la intrínseca con el saber y la trascendente concernida con el dar. De tal manera, los tres niveles pueden alcanzarse por medio de la actitud del docente con sus estudiantes. De esta manera, los tres niveles de motivación por parte del docente se ven ilustrados en los resultados obtenidos al aplicar los instrumentos, ya que los y las jóvenes consideran que los educadores son factor clave en la motivación (Carrasco y Baignol, 2004).

\section{Factores Académicos}

Los estudiantes señalan que es fundamental contar con material de apoyo para el estudio, lo que la Comisión Organizadora de las Olimpiadas debería de generar 
Factores que influyen en la participación y el desempeño académico de los estudiantes que compiten en la vi olimpiada costarricense de ciencias biológicas (OLICOCIBI).

José M. Pereira Chaves, Verónica Ulate Campos, Stephanie Arroyo Jiménez.

según los objetivos planteados en el temario, porque eso les permitiría prepararse de la mejor manera para poder realizar las pruebas y alcanzar mejores rendimientos académicos.

Se logró determinar que los estudiantes participantes muestran una mayor preferencia por la materia de Biología, que por las otras Ciencias (Física y Química), tal y como se evidencia en el figura 1. Esto es fundamentado por Villegas (2009), quién afirma que los estudiantes a nivel nacional presentan una tendencia a escoger Biología como ciencia para la realización de las pruebas de Bachillerato, puesto que prefieren evitar los cálculos y fórmulas que deben aplicar en las otras asignaturas. Los jóvenes afirman sentir un agrado sobre la temática desarrollada en Biología, ya que les permite contextualizar muchos contenidos. Es esta complacencia lo que los motiva a participar de este tipo de competencias cognitivas, como lo son las OLICOCIBI, ya que se estaría midiendo sus conocimientos en esa área tan afín a ellos.

Figura 1. Ciencia Natural de mayor preferencia por los estudiantes y profesores. I etapa, agosto 2013.

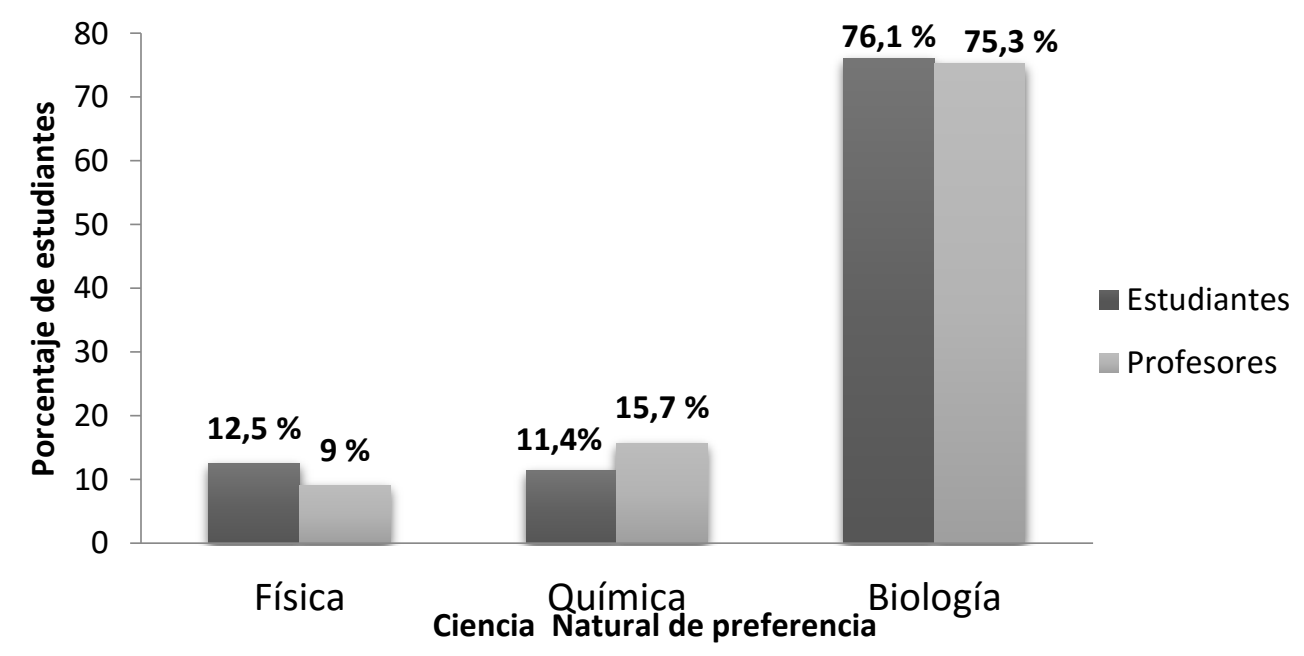

Fuente: Elaboración propia 
Tanto los docentes como los estudiantes, manifestaron más afinidad por las Ciencias de la vida. Este es un factor clave en el éxito académico de los estudiantes, ya que la identificación con esta ciencia genera mayor interés hacia la materia por ende podría ser un factor clave para la obtención de buenas calificaciones en las pruebas realizadas en las OLICOCIBI.

Por otro lado, el apoyo que los estudiantes reciben por parte del centro educativo es clave para lograr buenos resultados en las pruebas realizadas. Al analizar los porcentajes obtenidos el $78,7 \%$ de los estudiantes encuestados aseguran haber recibido algún tipo de apoyo por parte de la institución a la que pertenecen, pero no fue lo suficiente, ya que dejaron de lado las lecciones de preparación para las OLICOCIBI que son tan importantes, así como el apoyo emocional y económico. Además, los estudiantes externan que es malo el apoyo que reciben por parte de la Institución, para que se les dé preparación previa para los exámenes de las Olimpiadas. A partir de lo anterior, se determinó que la ayuda brindada por parte de la administración de la institución educativa, es considerada entre mala y regular, según lo expresan los estudiantes, ya que consideran que no es suficiente para la adecuada participación en eventos científicos. (Figura 2). 
Figura 2. Percepción de los estudiantes en cuanto al apoyo que reciben por parte de la Administración del Colegio para asistencia y participación en OLICOCIBI. I etapa, agosto 2013.

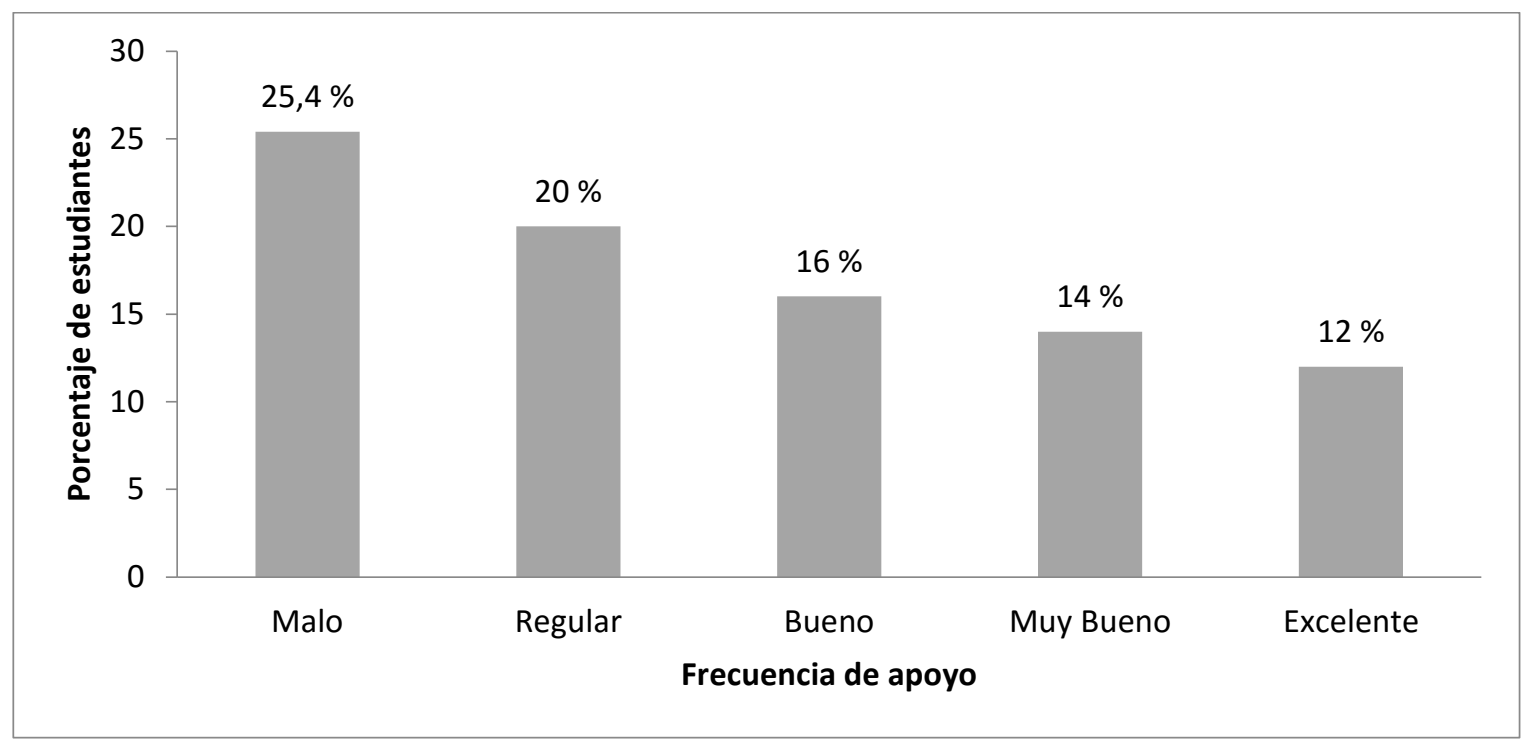

Fuente: Elaboración propia.

Se logró determinar que en la mayoría de instituciones públicas, a los docentes no se les asigna lecciones $u$ horas extras para que atiendan a los estudiantes que van a participar en las OLICOCIBI, contrario a otras instituciones privadas, donde al docente si se les conceden, dejando en desventaja a los estudiantes que no recibieron la ayuda de un docente.

\section{Factores Económicos}

El nivel socioeconómico que presentan las familias de los participantes repercute en el rendimiento académico y educativo alcanzado. Aunque el sistema educativo sea fácilmente accesible a todos, mediante la gratuidad o los sistemas de ayudas y becas, todavía en nuestra sociedad las facilidades económicas permiten un mayor acceso a los bienes culturales y educativos. Por otro lado, el nivel socioeconómico suele estar relacionado, aunque no siempre, con el nivel educativo y cultural de los padres (Legazpe, 2008). 
Al interpretar los resultados obtenidos mediante los instrumentos aplicados, se determinó que el total de estudiantes participantes en la categoría A provienen de centros educativos con diferentes modalidades como se ilustra a continuación:

\section{Cuadro 2: Modalidad de los Centros Educativos participantes en la VII OLICOCIBI, 2013.}

\begin{tabular}{l|l}
\hline Modalidad Académica del Centro Educativo & Porcentaje de participante \\
\hline Centros Educativos Privados & $33,0 \%$ \\
\hline Centros Educativos Académicos diurnos & $28,6 \%$ \\
\hline Técnicos Profesionales & $23,8 \%$ \\
\hline Colegios Científicos & $9,5 \%$ \\
\hline Experimental Bilingüe & $4,8 \%$ \\
\hline
\end{tabular}

Fuente: Elaboración propia.

Se pudo determinar que la eficacia demostrada por los estudiantes en las pruebas, está relacionada por la institución a la que pertenecen, y a la modalidad de dicho centro educativo, ya que algunas le dan mayor énfasis hacia algunas asignaturas en especial el área de las Ciencias, como es el caso de los Colegios Científicos y los Centros Educativos Privados. Según los resultados obtenidos en la aplicación de las encuestas, la mayoría de los estudiantes pertenecientes a estos centros educativos aseguran haber recibido tutorías y mayor apoyo por parte del docente para la participación.

El desempeño de los estudiantes se determina no sólo por las características individuales y los entornos familiares que condicionan el proceso de aprendizaje, sino por los recursos con los que cuentan las instituciones a las que asisten. El 
Factores que influyen en la participación y el desempeño académico de los estudiantes que compiten en la vi olimpiada costarricense de ciencias biológicas (OLICOCIBI).

José M. Pereira Chaves, Verónica Ulate Campos, Stephanie Arroyo Jiménez.

entorno familiar de los estudiantes explica sólo parte de las disparidades socioeconómicas en educación, la falta de ayudas del estado (becas) puede ser un factor clave para que los estudiantes progresen en sus estudios (Muñoz et al., 2009).

Los factores que influyen en el bajo rendimiento académico, estarían relacionados con los aspectos académicos, económicos y sociales de los estudiantes, además de los bajos niveles de: motivación, inteligencia, ingresos económicos, hábitos, estrategias y estilos de aprendizaje.

Según Tonconi (2010), los directores y profesores mencionan que los factores que inciden en el rendimiento académico escolar son los mismos que favorecen la calidad de la educación: el apoyo familiar, formación del profesor, material didáctico apropiado, currículo acorde a la realidad, infraestructura adecuada y la administración eficaz y los mismos coinciden con los instrumentos aplicados, tanto a docentes como estudiantes, en los cuales mencionan como clave el papel del docente en el proceso enseñanza-aprendizaje, así como los padres de familia y la institución educativa.

\section{Características del docente que Enseña Ciencias y que promueve la participación en actividades de carácter científico como lo es las OLICOCIBI.}

Según CONARE (2010), en su tercer Informe del Estado de la Educación muestra que en los últimos años se ha ido elevando el perfil de los docentes que tienen a cargo la formación científica en secundaria. De esta forma, al contar con mejores perfiles, la expectativa es ofertar una mejor formación científica en las instituciones de enseñanza media. Es de esperar entonces que en los próximos años se evidencie un nivel de rendimiento mayor en las pruebas de bachillerato, una mejor comprensión de los conceptos científicos y un mejor perfil de ingreso a los cursos básicos de Ciencias en las universidades. 
Según la información recopilada a través de los instrumentos aplicados a los profesores tutores, tanto en la fase preparatoria como en la etapa final y tomando en cuenta las características que se han presentado en los tres Informes del Estado de la Educación mencionados en el fundamento teórico, se determinó que las características del educador en Ciencias y que propicia la participación de sus educandos en eventos científicos son:

- Desarrolla giras educativas.

- Promueve la investigación científica.

- Promueve la alfabetización científica.

- Desarrolla experimentos sencillos en el aula.

- Causa que el estudiante auto dirija su aprendizaje.

- Contextualiza los conceptos que desarrolla en clase.

- Desarrolla diversas actividades de mediación en el aula.

- Le interesa estar participando en los distintos eventos científicos.

- Constantemente está facilitándole materiales de interés a sus estudiantes.

- Promueve la puesta en práctica de conocimientos durante el desarrollo de la clase.

El docente que imparte la asignatura de Ciencias en cualquiera de sus áreas (Física, Química y Biología) debe cumplir con ciertas características que son fundamentales para el buen desarrollo y cumplimiento de los objetivos propuestos en los programas de estudio, que van desde el promover la investigación y la alfabetización científica, hasta el desarrollo de experimentos, así como giras educativas. 
Factores que influyen en la participación y el desempeño académico de los estudiantes que compiten en la vi olimpiada costarricense de ciencias biológicas (OLICOCIBI).

José M. Pereira Chaves, Verónica Ulate Campos, Stephanie Arroyo Jiménez.

\section{Lineamientos a considerar en las futuras participaciones estudiantiles e institucionales.}

Considerando los instrumentos aplicados tanto a los docentes tutores como a los estudiantes participantes de la Categoría A, así como entrevistas estructuradas y no estructuradas aplicadas en la etapa de trabajo de campo, se recopilaron los siguientes lineamientos y recomendaciones, algunas tomadas textuales de los instrumentos aplicados

- La elaboración de material de apoyo por parte de la comisión organizadora que responda a los objetivos y contenidos de las pruebas a evaluar con el fin de lograr una mejor preparación.

- Capacitaciones en temas de Biología a docentes tutores con el fin de actualizarlos y brindarles diversos temas de interés que puedan presentar a los estudiantes, ya que como es bien sabido la formación docente es la clave para alcanzar una educación de calidad en las instituciones educativas.

- Capacitación o curso de preparación a los estudiantes participantes para enfrentar los exámenes con un mejor fundamento por parte de la Comisión organizadora de OLICOCIBI.

- Mayor apoyo a los estudiantes que compiten por parte de las Instituciones Educativas tanto económico, académico y emocional; con el fin de alcanzar un mejor rendimiento en la competencia.

- Capacitar a los estudiantes que clasificaron a la segunda etapa en elaboración de informes y artículos científicos claves en la etapa de trabajo de campo por parte de la Comisión Organizadora.

- Trabajo en equipo por parte de los docentes tutores y los estudiantes participantes en la segunda etapa (fase final) específicamente en el trabajo de campo y la elaboración de informes.

Esta investigación tuvo como fin, identificar y reconocer la existencia de posibles factores que intervienen en el éxito de los estudiantes en la OLICOCIBI, los 
factores que se pudieron determinar fueron motivacionales, como el apoyo que reciben los estudiantes de sus docentes, centros educativos, así como de sus familiares; académicos como la afinidad de los estudiantes y los docentes por la asignatura de biología, la preparación previa a la OLICICIBI, en algunos casos sin centros de estudio por parte de los docente tutores, el poco apoyo que recibieron por parte de la institución a la que pertenecen y un punto clave la formación del docente a cargo; económicos como el costear pasajes, alimentación y algunos casos hospedaje para la participación, tanto de la etapa eliminatoria como de la fase final sin el apoyo del centro educativo, y a veces sin el de los padres de familia que no se encuentran en la capacidad de costear gastos de esa índole.

El identificar los factores que influyen en OLICOCIBI es una forma de fortalecer el programa, ya que uno de los objetivos de esta investigación es brindar recomendaciones a la Comisión Organizadora con el fin de mejorar y fortalecer las debilidades que se presenten, así como tomar en consideración las sugerencias que tanto estudiantes como docentes e investigadoras puedan dar al mismo.

Los estudiantes y docentes que han participado de competencias cognitivas, como OLICOCIBI, afirman que son una alternativa innovadora para complementar los aprendizajes del aula, el espíritu de competitividad que los educandos muestran, así como ese deseo de superación, indican al educador participante que existen formas diferentes de enseñar y de aprender, que no solo de la manera tradicional se puede enseñar.

\section{Reflexiones Conclusivas}

Se determinaron los principales factores motivacionales, económicos y académicos que influyen directamente en la participación y el rendimiento académico de la competencia por tanto. 
Factores que influyen en la participación y el desempeño académico de los estudiantes que compiten en la vi olimpiada costarricense de ciencias biológicas (OLICOCIBI).

José M. Pereira Chaves, Verónica Ulate Campos, Stephanie Arroyo Jiménez.

\section{Motivacionales}

- El docente tutor es generador de la motivación, quien es el motivo de participación de los estudiantes porque es quien los incentiva a participar y les apoya mediante explicaciones y material didáctico. Además el apoyo de los padres de familia y compañeros es clave para que ellos puedan alcanzar buenos resultados, ya que, según los resultados obtenidos, el apoyo familiar es poco.

- Con base en los factores académicos, se identificó mayor preferencia por la materia de biología que otras ciencias, y motivado por la situación de evitar los cálculos y fórmulas que deben aplicar en las otras asignaturas.

- Otro factor académico, es la ayuda brindada por parte de las instituciones educativas, que es considerada entre mala y regular, porque no se les asignaron lecciones adicionales a los docentes tutores para la preparación de los jóvenes en estas justas.

\section{Económicos:}

- Los estudiantes requieren apoyo económico por parte de los Centros Educativos a los que pertenecen para sufragar gastos de transporte, alimentación, materiales, y hospedaje en algunos casos, entre otros elementos.

- Entre los factores económicos, se recopiló información de que algunos estudiantes no tienen una vivienda propia, generando salidas de ingresos considerables por parte de los jefes de hogar, lo que los limita económicamente a sufragar gastos varios en cuanto a la educación de sus hijos.

\section{Formación docente:}

- El docente que enseña Ciencias debe cumplir con una serie de características claves, con el fin de ir mejorando el perfil de los educadores que tienen a cargo la formación científica en secundaria ya que al contar con mejores 
Factores que influyen en la participación y el desempeño académico de los estudiantes que compiten en la vi olimpiada costarricense de ciencias biológicas (OLICOCIBI).

José M. Pereira Chaves, Verónica Ulate Campos, Stephanie Arroyo Jiménez.

perfiles, la expectativa es que se debe ofrecer una mejor formación científica en las instituciones de enseñanza media.

- El docente que suscita la participación en eventos científicos como competencias cognitivas es el que promueve la alfabetización y la investigación científica, el que contextualiza contenidos y siempre está tratando de innovar y buscar nuevas opciones para mejorar su labor día con día.

\section{Referencias}

Álvarez, R. (1998). Debates Actuales Sobre La Pedagogía. Cuba, La Habana.

Carrasco, J. y Baignol, J. (2004). Técnicas y Recursos para Motivar a los Alumnos. Sexta Edición. Madrid. España: Editorial RIALP S.A. Pp. 40 - 44.

Camacho, S. (2011). Aportes del proyecto OLICOCIBI en la alfabetización científica de sus participantes en sus dimensiones conceptual, procedimental y actitudinal. Tesis Licenciatura. Universidad Nacional, Heredia, Costa Rica. P.6, 16.

CONARE (2010). Tercer Informe del Estado de la Educación / Consejo Nacional de Rectores. (3 ed.). San José Costa Rica. Pp.36, 37, 38.

Comisión Organizadora de la Olimpiada Costarricense de Ciencias Biológicas. (2009). Normativa de las Olimpiadas Costarricenses de Ciencias Biológicas. Universidad Nacional. Heredia. Costa Rica.

Lafrancesco, G. (2005). Didáctica de la Biología aportes a su desarrollo. Primera edición. Bogotá, Colombia: Cooperativa Editorial Magisterio.. p 75, 76.

Legazpe, F. (2008). Motivar para el aprendizaje desde la actividad orientadora. Ministerio de Educación y Ciencia. Secretaria General de Educación. OMAGRAF, S.L. 2008. p. 17, 20, 21, 22, 135,144.

Mora, R. y Calderón, M. (2008). Colegio Científico de Pérez Zeledón Quince años al Servicio de Costa Rica. Primera Edición. San José, Costa Rica: Publicaciones El Atabal, S.A. p.135-137.

Muñóz, C., Márquez, A., Sandoval, A. y Sánchez, H. (2004). Factores Externos e Internos a las Escuelas que Influyen en el Logro Académico de los 
Factores que influyen en la participación y el desempeño académico de los estudiantes que compiten en la vi olimpiada costarricense de ciencias biológicas (OLICOCIBI).

José M. Pereira Chaves, Verónica Ulate Campos, Stephanie Arroyo Jiménez.

estudiantes de Nivel Primaria en México, 1998-2002. Análisis Comparativo entre entidades con diferente nivel de desarrollo. Ciudad de México: Instituto de Investigaciones para el Desarrollo de la Educación. Universidad Iberoamericana. p.2, 6.

Orozco, E. (2011). El Educador en su quehacer Motivacional a favor del educando, dentro de su actividad docente. Congreso Didáctico. Ponencias presentadas por cohorte 2011. Para el curso de didáctica de la enseñanza superior. Universidad de San Carlos de Guatemala. p. 27, 28.

Perales, J.P. y Cañal, P. (2000). Didáctica de las Ciencias experimentales. España: Editorial Marfil, S.A. ALCOY. Pp. 451, 453 y 466.

Pereira, J. (2010). La enseñanza de la Biología en Costa Rica los retos del abordaje pedagógico para la alfabetización científica y la motivación estudiantil en secundaria. Congreso Iberoamericano de Educación. Buenos Aires Argentina. p.3.

Pereira, J., Camacho, S. y Muñoz, N. (2012). Informe Final de Proyecto Olimpiada Costarricense de Biología (030303), 2007-2011. Universidad Nacional. Heredia, Costa Rica. p.4.

Rodríguez, G., Gil, J. y García, E. (1996) Metodología de la Investigación Cualitativa. Málaga: Aljibe. España. p.3, 4, 9.

Tonconi, J. (2010). Factores que influyen en el rendimiento académico y la deserción de los estudiantes de la facultad de ingeniería económica de la una-puno, periodo 2009. Cuadernos de Educación y Desarrollo, 2(1), 23. p.1.

Villegas, J. (2009). Examen de bachillerato de Biología seduce a estudiantes. En La Nación. (San José, Costa Rica), 06 de noviembre, 2009. p.107, 108.

Veglia, S. (2007). Ciencias Naturales y Aprendizaje Significativo, Claves para la reflexión didáctica y la planificación. Primera Edición. Buenos Aires, Argentina: Ediciones Novedades Educativas. 